\title{
An Equivalence Principle for OFDM-Based Combined Bulk/Per-Subcarrier Relay Selection over Equally Spatially Correlated Channels
}

\author{
Shuping Dang, Student Member, IEEE, and Justin P. Coon, Senior Member, IEEE, Gaojie Chen, Member, IEEE
}

\begin{abstract}
In this paper, we propose a novel relay selection scheme for orthogonal frequency-division multiplexing (OFDM) systems by combining conventional bulk and per-subcarrier selection schemes, and analyze its outage performance over equally spatially correlated channels. Specifically, the combined selection scheme selects only two relays at the first attempt and performs per-subcarrier selection over these two relays. We analyze the asymptotic outage performance of the combined selection scheme in the high signal-to-noise (SNR) region, and prove a generalized theorem. This theorem states that the combined selection can achieve an optimal outage probability equivalent to the persubcarrier selection at high SNR without using the full set of available relays for selection. This unique property is termed the equivalence principle, and it holds for all correlation conditions. To explore this principle, we consider three examples: decodeand-forward (DF), fixed-gain (FG) amplify-and-forward (AF) and variable-gain (VG) AF relay systems. Furthermore, two extended applications, antenna selection and branch selection, are also considered to reveal the feasibility and the expandability of the equivalence principle. Our analysis is verified by Monte Carlo simulations. The proposed combined selection and the proved theorem provide a general and feasible solution to the trade-off between system complexity and outage performance when relay selection is applied.
\end{abstract}

Index Terms-Combined selection, relay selection, channel correlation, OFDM, asymptotic analysis.

\section{INTRODUCTION}

$\mathbf{S}$ INCE the proposal of combined bulk/per-subcarrier selection designed specifically for transmit antenna selection, combined selection has shown its excellent properties in terms of reducing system complexity and obtaining optimal outage performance [1]. Meanwhile, the cooperative network, as an important concept in the field of communication engineering, has been proposed and analyzed for decades [2]-[4]. With the development of cooperative networks, the combined selection scheme is regarded as an effective method to reduce the system complexity and simultaneously obtain the optimal performance at high signal-to-noise ratio (SNR) for orthogonal frequencydivision multiplexing (OFDM) relay systems [5]. By the combined bulk/per-subcarrier selection, only two out of the total

(C)2015 IEEE. Personal use of this material is permitted. However, permission to use this material for any other purposes must be obtained from the IEEE by sending a request to pubs-permissions@ieee.org.

This work was supported by the FP7 DIWINE project (Grant Agreement CNET-ICT-318177) and the grant from China Scholarship Council (No. 201508060323).

The authors are with the Department of Engineering Science, University of Oxford, Parks Road, Oxford, UK, OX1 3PJ; tel: +44 (0)1865 283 393, (e-mail: \{shuping.dang, justin.coon, gaojie.chen\}@eng.ox.ac.uk). available relays are selected according to a certain criterion and the per-subcarrier selection is performed over these two relays for all subcarriers individually ${ }^{1}$. Its applicability in cooperative networks over independent and identically distributed (i.i.d.) channels is numerically analyzed in [9]. Also, a practical implementation scheme of combined selection is proposed in [10] and a comprehensive comparison among these three selection schemes in super dense networks are provided in [11]. To be more clear, the proposed combined selection scheme as well as the conventional bulk and per-subcarrier selection schemes are illustrated in Fig. 1.

Most previous works on combined relay selection assume i.i.d. fading channels, which are practically rare owing to the insufficient physical separations among relays [12]. The performance analysis of combined selection over spatially correlated fading channels is still an open issue. By literature review, a number of relevant correlated channel models can be considered. A comprehensive analysis of outage probability of multi-branch selection over spatially correlated fading channels was reported in [13]. A triple channel correlation scenario is considered in [14], but it cannot be applied to a general multi-branch case with an arbitrary number of parallel branches. The most useful and relevant spatially correlated channel model for our study is given in [15], in which a set of channel gains produced by equally correlated channels can be transfered to a set of conditionally independent channel gains. Employing this model, we are able to analyze the outage performance using conventional analytical tools, e.g. order statistics. Therefore, in this paper, we employ the correlation model proposed in [15] to analyze a general OFDM system applying combined relay selection.

It should be noted that we only consider the equally spatial correlation among channels within the same hop and still maintain the i.i.d. assumption between the channels in two hops, which is different from the cross-hop correlation scenario presented in [16]. This is simply because we assume all relays are physically stationary over a coherent time interval, so that the correlation produced by Doppler shift between two hops is negligible [16]. We assume a block fading model in frequency akin to systems that employ a resource block frame/packet structure (e.g. LTE), and hence the i.i.d. assumption in frequency holds. The identical distri-

\footnotetext{
${ }^{1}$ Note that, in this paper the combined relay selection employed is different from the multi-relay selection schemes proposed in [6]-[8]. In particular, each subcarrier is only forwarded by one relay, and all transmissions corresponding to all subcarriers are carried out simultaneously.
} 


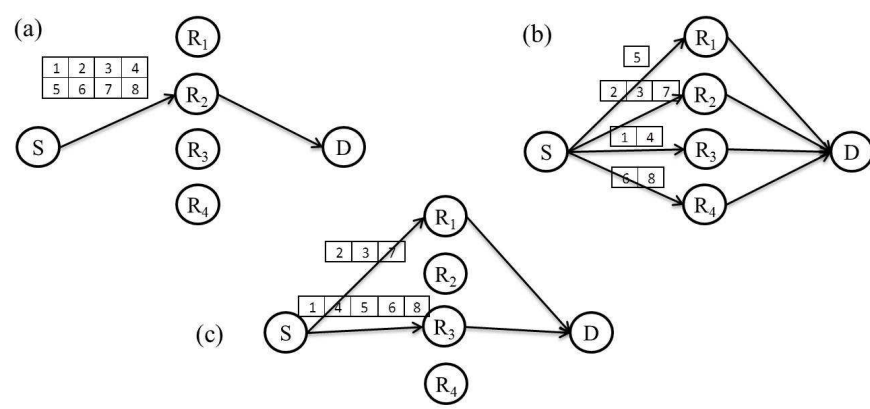

Fig. 1. Illustration of (a) bulk, (b) per-subcarrier and (c) combined bulk/persubcarrier relay selection schemes for single source, single destination and multiple relays, given $K=8, M=4$ and $L=2$. The numbers in boxes are corresponding to the sequence numbers of subcarriers.

bution assumption in the same hop is supported by the fact that relays are normally aggregated within a region with a small radius compared to the distance between source and destination (a.k.a. relay cluster) [17], [18], and this refers to the widely applied non-independent and identically distributed channel model fading channel model [19], [20].

Overall, the main contributions of this paper are summarized as follows:

- We propose and prove a generalized theorem stating that the combined selection will always be able to achieve an optimal outage performance as per-subcarrier selection at high SNR over spatially correlated channels, as long as certain general conditions can be provided. In this paper, we refer to this asymptotic behavior as the equivalence principle.

- We provide a generic asymptotic expression for outage probability at high SNR when applying combined selection.

- We show that this asymptotic expression can be easily applied in a variety of selection scenarios for different relays, namely, decode-and-forward (DF) relay system, fixed-gain (FG) amplify-and-forward (AF) relay system and variable-gain (VG) AF relay systems. Meanwhile, we consider two extended applications different from relay selections in order to reveal the feasibility and the expandability of the equivalence principle.

The rest of the paper is organized as follows. Section II gives the system model. Then, the asymptotic outage performance of combined selection over spatially correlated channels is generally analyzed and compared to the outage performance of per-subcarrier selection in Section III. Also, based on this general analysis, three relay applications are discussed in depth in Section IV. Furthermore, two extended applications are briefly considered in Section V. After that, numerical simulations are carried out, which verify our analysis in Section VI. Finally, the paper is concluded in Section VII.

\section{SYSTEM MODEL}

\section{A. System configurations}

We consider a typical two-hop OFDM system with $K$ orthogonal subcarriers and $M$ relays from the single source to the single destination. Hence, $M K$ channels in total are constructed at each hop and for a given subcarrier $k$, the $M$ channels are equally correlated in space. For the $m$ th relay, the end-to-end SNR transmitted on the $k$ th subcarrier is denoted by $\operatorname{SNR}(m, k), \forall m \in \mathcal{M}=\{1,2, \cdots, M\}$ and $\forall k \in \mathcal{K}=\{1,2, \cdots, K\}$. Accordingly, the a priori outage probability without conditioning on any selection can be defined as

$$
F(s)=\mathbb{P}\{\operatorname{SNR}(m, k)<s\},
$$

where $s$ is the end-to-end SNR threshold; $\mathbb{P}(\cdot)$ denotes the probability of the enclosed.

For a two-hop system, there are $M$ channels for a given subcarrier in each hop, denoted as $h_{i}(m, k), i \in\{1,2\}$. We assume that the channels are mutually correlated with the common cross-correlation coefficient denoted as $\rho_{i}\left(0 \leq \rho_{i} \leq 1\right)$. Therefore, we can construct the equally correlated Rayleigh fading channel by [15]

$$
\begin{aligned}
h_{i}(m, k) & =\left[\sqrt{1-\rho_{i}} x_{i}(m, k)+\sqrt{\rho_{i}} x_{i 0}(k)\right] \\
& +j\left[\sqrt{1-\rho_{i}} y_{i}(m, k)+\sqrt{\rho_{i}} y_{i 0}(k)\right],
\end{aligned}
$$

where $j=\sqrt{-1} ; x_{i}(m, k), y_{i}(m, k) \sim \mathcal{N}\left(0, \mu_{i} / 2\right)$ are i.i.d. and $\mu_{i}$ is the average channel gain at each hop; $x_{i 0}(k), y_{i 0}(k) \sim \mathcal{N}\left(0, \mu_{i} / 2\right)$ are i.i.d. and serve as references to correlate all channels. Hence, $\forall m \neq n$ we have $\mathbb{E}\left\{h_{i}(m, k) h_{i}^{*}(n, k)\right\} / \sqrt{\mathbb{E}\left\{\left|h_{i}(m, k)\right|^{2}\right\} \mathbb{E}\left\{\left|h_{i}(n, k)\right|^{2}\right\}}=\rho_{i}$. Also, by the fundamental theory of statistics, we have $h_{i}(m, k) \sim \mathcal{C N}\left(0, \mu_{i}\right)$. As a result, $\left|h_{i}(m, k)\right|^{2} \sim \chi_{2}\left(0, \mu_{i}\right)$. Meanwhile, how both $h_{1}(m, k)$ and $h_{2}(m, k)$ are organized in $\operatorname{SNR}(m, k)$ depends on the adopted forwarding protocol, i.e. DF, FG AF and VG AF relaying protocols. Specific relations among $\operatorname{SNR}(m, k), h_{1}(m, k)$ and $h_{2}(m, k)$ are detailed in Section IV.

Besides, we assume that the channel state information (CSI) is perfectly estimated and shared among all communication nodes $^{2}$, and the relaying network operates in a half-duplex protocol so that two orthogonal time slots are required for one complete transmission from source to destination. All noise statistics are i.i.d. zero-mean, complex Gaussian (ZMCG) random variables with variance $N_{0} / 2$ per dimension. Meanwhile, we further suppose that equal bit and power allocation schemes are applied, so that the average transmit power per subcarrier at the source and at each utilized relay is denoted by $P_{t}$.

\section{B. Selection schemes}

1) Combined selection: As a compromise selection scheme between bulk and per-subcarrier selections, combined selection scheme first selects two relays according to the criterion

$$
\mathcal{L}_{\text {comb }}=\arg \max _{\mathcal{L}_{2} \subseteq \mathcal{M}} \min _{k \in \mathcal{K}} \max _{m \in \mathcal{L}_{2}} \operatorname{SNR}(m, k),
$$

\footnotetext{
${ }^{2}$ The CSI is usually estimated through pilots and feedback (e.g. [21]) and the CSI estimation without feedback may also be applied (e.g. [22]).
} 
where $\mathcal{L}_{2}$ identifies a pair of relays that can be employed to carry out per-subcarrier selection and $\left|\mathcal{L}_{2}\right|=2^{3}$; Obviously, we have $M(M-1) / 2$ available options of $\mathcal{L}_{2}$ in total.

After that, per-subcarrier selection is performed over the two relays in $\mathcal{L}_{\text {comb }}$ in a per-subcarrier manner by

$$
l_{\text {comb }}(k)=\arg \max _{l \in \mathcal{L}_{\text {comb }}} \operatorname{SNR}(l, k) .
$$

Therefore, when combined selection is employed, the $a$ posteriori outage probability depending on selection can be defined as

$$
F_{\text {comb }}(s)=\mathbb{P}\left\{\min _{k \in \mathcal{K}} \max _{l \in \mathcal{L}_{\text {comb }}} \operatorname{SNR}(l, k)<s\right\} .
$$

2) Per-subcarrier selection: Per-subcarrier selection scheme selects multiple relays (up to $K$ ) from $M$ relays in a per-subcarrier manner so that all subcarriers can be forwarded via their optimal relays and thus the optimal outage performance is attainable. For the $k$ th subcarrier, the selection criterion is thereby

$$
L_{p s}(k)=\arg \max _{m \in \mathcal{M}} \operatorname{SNR}(m, k) .
$$

Therefore, when per-subcarrier selection is employed, the $a$ posteriori outage probability can be defined as

$$
F_{p s}(s)=\mathbb{P}\left\{\min _{k \in \mathcal{K}} \max _{m \in \mathcal{M}} \operatorname{SNR}(m, k)<s\right\} .
$$

Although there exist three commonly used relay selection schemes for OFDM systems, the equivalence principle potentially exists between combined and per-subcarrier selections only [1]. Therefore, we only analyze and compare the outage performances of per-subcarrier and combined selections in the rest of this paper.

\section{Outage Performance Analysis}

\section{A. Conditionally independent variable transformation}

As detailed in [15], we take a similar approach to transform a set of equally correlated random variables to a set of conditionally independent random variables, so that conventional analytical tools, e.g. order statistics can be applied to analyze them effectively. To do so, we first assume that two references for each subcarrier $x_{i 0}(k)$ and $y_{i 0}(k)$ are fixed and have $x_{i 0}(k)=X_{i 0}(k)$ and $y_{i 0}(k)=$ $Y_{i 0}(k)$. Therefore, the conditional distribution of $h_{i}(m, k)$ is $\mathcal{C N}\left(\sqrt{\rho_{i}}\left[X_{i 0}(k)+j Y_{i 0}(k)\right], \mu_{i}\left(1-\rho_{i}\right)\right)$. Consequently, given $x_{i 0}(k)=X_{i 0}(k)$ and $y_{i 0}(k)=Y_{i 0}(k),\left|h_{i}(m, k)\right|^{2} \sim$ $\chi_{2}\left(\sqrt{\rho_{i}\left[X_{i 0}^{2}(k)+Y_{i 0}^{2}(k)\right]}, \mu_{i}\left(1-\rho_{i}\right)\right)$. If we denote $T_{i}(k)=$ $X_{i 0}^{2}(k)+Y_{i 0}^{2}(k)$, the conditional probability density function (PDF) and the conditional cumulative distribution function (CDF) of $\left|h_{i}(m, k)\right|^{2}$ are given by [23]

$$
f_{h_{i}}\left(s \mid T_{i}(k)\right)=\frac{1}{\mu_{i}\left(1-\rho_{i}\right)} e^{-\frac{s+\rho_{i} T_{i}(k)}{\mu_{i}\left(1-\rho_{i}\right)}} I_{0}\left(\frac{2 \sqrt{\rho_{i} T_{i}(k) s}}{\mu_{i}\left(1-\rho_{i}\right)}\right)
$$

\footnotetext{
${ }^{3}$ We can alternatively employ a three-relay subset $\mathcal{L}_{3}$ or even a $(M-1)$ relay subset $\mathcal{L}_{M-1}$ in this step. All cases will be the same at high SNR, just with a different convergence rate. However, $\mathcal{L}_{2}$ is the most representative case, since it is the closest to bulk selection. Therefore, we can later show that as long as one more relay is selected compared to bulk selection (the worst case), the outage performance given by combined selection is asymptotic to the per-subcarrier selection's (the best case).
}

and

$$
F_{h_{i}}\left(s \mid T_{i}(k)\right)=1-Q\left(\sqrt{\frac{2 \rho_{i} T_{i}(k)}{\mu_{i}\left(1-\rho_{i}\right)}}, \sqrt{\frac{2 s}{\mu_{i}\left(1-\rho_{i}\right)}}\right),
$$

where $I_{0}(\cdot)$ is the zero order modified Bessel function of the first kind; $Q(\cdot, \cdot)$ is the first order Marcum $\mathrm{Q}$ function.

Meanwhile, the PDF and CDF of $T_{i}(k)$ can be obtained as:

$f_{T_{i}}\left(T_{i}(k)\right)=\frac{1}{\mu_{i}} e^{-\frac{T_{i}}{\mu_{i}}} \Leftrightarrow F_{T_{i}(k)}\left(T_{i}(k)\right)=1-e^{-\frac{T_{i}(k)}{\mu_{i}}}$.

Denote $\mathbf{T}_{1}=\left\{T_{1}(1), T_{1}(2), \ldots, T_{1}(K)\right\}$ and $\mathbf{T}_{2}=$ $\left\{T_{2}(1), T_{2}(2), \ldots, T_{2}(K)\right\}$. Because $\forall i \in\{1,2\}$ and $k \in \mathcal{K}$, $T_{i}(k)$ are mutually independent, we thereby can derive the joint PDF corresponding to $\mathbf{T}_{1}$ and $\mathbf{T}_{2}$ by

$$
f_{\mathbf{T}}\left(\mathbf{T}_{1}, \mathbf{T}_{2}\right)=\left(\frac{1}{\mu_{1} \mu_{2}}\right)^{K} \prod_{k=1}^{K}\left(e^{-\frac{T_{1}(k)}{\mu_{1}}} e^{-\frac{T_{2}(k)}{\mu_{2}}}\right) .
$$

Accordingly, we can denote the conditional a priori outage probability as $F\left(s \mid T_{1}(k), T_{2}(k)\right)$. We can also denote the conditional a posteriori outage probabilities for combined selection and per-subcarrier selection as $F_{c o m b}\left(s \mid \mathbf{T}_{1}, \mathbf{T}_{2}\right)$ and $F_{p s}\left(s \mid \mathbf{T}_{1}, \mathbf{T}_{2}\right)$ respectively.

\section{B. Asymptotic outage performance: main results}

We present the all-important contribution of this paper here. A generalized equivalence principle can be stated as follows.

Theorem 1: If the conditional CDF of the end-to-end SNR, $F\left(s \mid T_{1}(k), T_{2}(k)\right)$, can be expanded in the variable $\bar{\gamma}=P_{t} / N_{0}$ as

$$
\begin{aligned}
F\left(s \mid T_{1}(k), T_{2}(k)\right) & =\sum_{i=i_{0}}^{\infty} c_{i}\left(s \mid T_{1}(k), T_{2}(k)\right)\left(\frac{1}{\bar{\gamma}}\right)^{\frac{i}{\theta}}[\ln (\bar{\gamma})]^{r} \\
& \sim c_{i_{0}}\left(s \mid T_{1}(k), T_{2}(k)\right)\left(\frac{1}{\bar{\gamma}}\right)^{\frac{i_{0}}{\theta}}[\ln (\bar{\gamma})]^{r},
\end{aligned}
$$

where $i_{0}$ is an integer given by $i_{0}=$ $\arg \min _{n \in \mathbb{N}}\left\{c_{n}\left(s \mid T_{1}(k), T_{2}(k)\right) \neq 0\right\} ; \quad \theta \quad$ is a nonzero natural number; $\left\{c_{i}\left(s \mid T_{1}(k), T_{2}(k)\right)\right\}$ represents a series of functions of $s$, given $T_{1}(k), T_{2}(k) ; r \in \mathbb{N}$, then combined selection is able to achieve an outage probability equivalent to conventional per-subcarrier selection as $\bar{\gamma} \rightarrow \infty$.

Proof: This is a powerful theorem not only suiting twohop relay selection, but also applying to transmit antenna selection and multi-hop branch selection by a slight modification (See Section V). We will show the proof of this theorem step by step in the following subsections.

1) Combined selection: Similar to Lemma 1 proposed in [1], we here propose and prove a congeneric lemma for the a posteriori outage performance of combined selection in the high SNR region for relay systems, which is also valid over correlated channels.

Lemma 1: Consider a generic two-hop OFDM system performing combined selection over $K$ orthogonal subcarriers and $M$ relays. By applying combined relay selection scheme, the worst possible end-to-end SNR has at least the $M$ th smallest value out of the total $M K$ end-to-end SNRs. 
Proof: We can take exactly the same step as given in [1] for the proof of the first part of Lemma 1 in that paper, but only vary the term 'channel gain' considered in multiple-input and multiple-output (MIMO) systems to 'end-to-end SNR' considered in two-hop cooperative systems. The validity of the proof still holds for this congeneric lemma, because the internal logic of this lemma does not change when considering different diversity systems.

From Lemma 1, it is clear that once an outage event occurs, there must exist at least one subcarrier on which all $M$ endto-end SNRs via $M$ relays drop below the outage threshold. Also, it is well known that the outage event is dominated by the deepest fade at high SNR and is asymptotic to the case where only one subcarrier on which all $M$ end-to-end SNRs via $M$ relays drop below the outage threshold at high SNR [24]. Consequently, we have

$$
\begin{aligned}
F_{\text {comb }}\left(s \mid \mathbf{T}_{1}, \mathbf{T}_{2}\right)= & \sum_{k=1}^{K}\left[F\left(s \mid T_{1}(k), T_{2}(k)\right)\right]^{M} \\
& +O\left(\left[F\left(s \mid T_{1}(k), T_{2}(k)\right)\right]^{M+1}\right) .
\end{aligned}
$$

Therefore, according to (12), the asymptotic expression for $F_{\text {comb }}\left(s \mid \mathbf{T}_{1}, \mathbf{T}_{2}\right)$ at $\bar{\gamma} \rightarrow \infty$ can be determined by

$$
\begin{aligned}
& F_{\text {comb }}\left(s \mid \mathbf{T}_{1}, \mathbf{T}_{2}\right) \\
& \quad \sim \sum_{k=1}^{K}\left\{c_{i_{0}}\left(s \mid T_{1}(k), T_{2}(k)\right)\left(\frac{1}{\bar{\gamma}}\right)^{\frac{i_{0}}{\theta}}[\ln (\bar{\gamma})]^{r}\right\}^{M} .
\end{aligned}
$$

2) Per-subcarrier selection: Similar to the derivation performed for combined selection, according to (7), the conditional outage probability of per-subcarrier selection can be asymptotically expressed by [25]

$$
\begin{aligned}
& F_{p s}\left(s \mid \mathbf{T}_{1}, \mathbf{T}_{2}\right)=1-\prod_{k=1}^{K}\left\{1-\left[F\left(s \mid T_{1}(k), T_{2}(k)\right)\right]^{M}\right\} \\
& \sim \sum_{k=1}^{K}\left\{c_{i_{0}}\left(s \mid T_{1}(k), T_{2}(k)\right)\left(\frac{1}{\bar{\gamma}}\right)^{\frac{i_{0}}{\theta}}[\ln (\bar{\gamma})]^{r}\right\}^{M} .
\end{aligned}
$$

3) Unconditional outage performance: Comparing the same asymptotic expressions given in (14) and (15), it is clear that when both multiply (11) and are integrated by $\mathbf{T}_{1}$ and $\mathbf{T}_{2}$ from zero to infinity, they will produce the same asymptotic outage performance as

$$
\begin{aligned}
& \left\{F_{\text {comb }}(s), F_{p s}(s)\right\} \sim K\left(\frac{1}{\mu_{1} \mu_{2}}\right)\left\{\left(\frac{1}{\bar{\gamma}}\right)^{\frac{i_{0}}{\theta}}[\ln (\bar{\gamma})]^{r}\right\}^{M} \\
& \times \int_{0}^{\infty} \int_{0}^{\infty}\left\{c_{i_{0}}\left(s \mid T_{1}, T_{2}\right)\right\}^{M}\left(e^{-\frac{T_{1}}{\mu_{1}}} e^{-\frac{T_{2}}{\mu_{2}}}\right) \mathrm{d} T_{1} \mathrm{~d} T_{2} .
\end{aligned}
$$

By (16), Theorem 1 is proved.

\section{Asymptotic Outage Performance Analysis WITH RELAY SELECTION}

\section{A. DF relay selection}

According to [26], the equivalent instantaneous end-to-end $S N R^{4}$ corresponding to the $k$ th subcarrier and the $m$ th relay using a DF protocol can be expressed as

$$
\operatorname{SNR}(m, k)=\frac{P_{t}}{N_{0}} \min \left(\left|h_{1}(m, k)\right|^{2},\left|h_{2}(m, k)\right|^{2}\right) .
$$

For brevity, denote $\psi_{i}=\mu_{i}\left(1-\rho_{i}\right) / 2$ and $\gamma_{i}=\left|h_{i}(m, k)\right|^{2}$. By series expansion, we can determine the asymptotic expression for the conditional CDF of the equivalent instantaneous end-to-end SNR for the $k$ th subcarrier at high SNR:

$F\left(s \mid T_{1}(k), T_{2}(k)\right) \sim\left[\frac{s}{2}\left(e^{-\frac{\rho_{1} T_{1}(k)}{2 \psi_{1}}} / \psi_{1}+e^{-\frac{\rho_{2} T_{2}(k)}{2 \psi_{2}}} / \psi_{2}\right)\right] \frac{1}{\bar{\gamma}}$.

Proof: See Appendix A.

Hence, by (12), $c_{i_{0}}\left(s \mid T_{1}(k), T_{2}(k)\right)$ can be determined by

$c_{i_{0}}\left(s \mid T_{1}(k), T_{2}(k)\right)=\frac{s}{2}\left(e^{-\frac{\rho_{1} T_{1}(k)}{2 \psi_{1}}} / \psi_{1}+e^{-\frac{\rho_{2} T_{2}(k)}{2 \psi_{2}}} / \psi_{2}\right)$.

Then, by (16), the unconditional outage probabilities for both selection schemes are determined by

$$
\begin{aligned}
\left\{F_{c o m b}(s), F_{p s}(s)\right\} & \sim K\left(1-\rho_{1}\right)\left(1-\rho_{2}\right)\left(\frac{s}{2 \bar{\gamma}}\right)^{M} \sum_{m=0}^{M}\left(\begin{array}{c}
M \\
m
\end{array}\right) \\
& \times \frac{\left(\frac{1}{\psi_{1}}\right)^{M-m}\left(\frac{1}{\psi_{2}}\right)^{m}}{\left[1+\rho_{1}(M-m-1)\right]\left[1+\rho_{2}(m-1)\right]} .
\end{aligned}
$$

\section{B. $F G$ AF relay selection}

It is also well known that for FG AF relay system, the instantaneous end-to-end SNR can be expressed as [27]

$$
\operatorname{SNR}(m, k)=\frac{\left|h_{1}(m, k)\right|^{2}\left|h_{2}(m, k)\right|^{2} P_{t}^{2}}{\left(\mu_{1} P_{t}+\left|h_{2}(m, k)\right|^{2} P_{t}+N_{0}\right) N_{0}} .
$$

By (8) and (9), we can derive the asymptotic expression for the conditional $\mathrm{CDF}$ at $\bar{\gamma} \rightarrow \infty$ by

$$
F\left(s \mid T_{1}(k), T_{2}(k)\right) \sim\left(\frac{\mu_{1} s}{4 \psi_{1} \psi_{2}} e^{-\frac{\rho_{1} T_{1}(k)}{2 \psi_{1}}} e^{-\frac{\rho_{2} T_{2}(k)}{2 \psi_{2}}}\right) \frac{\ln (\bar{\gamma})}{\bar{\gamma}} .
$$

Proof: See Appendix B.

Consequently, $c_{i_{0}}\left(s \mid T_{1}(k), T_{2}(k)\right)$ is given by

$$
c_{i_{0}}\left(s \mid T_{1}(k), T_{2}(k)\right)=\frac{\mu_{1} s}{4 \psi_{1} \psi_{2}} e^{-\frac{\rho_{1} T_{1}(k)}{2 \psi_{1}}} e^{-\frac{\rho_{2} T_{2}(k)}{2 \psi_{2}}} .
$$

Therefore, according to (16), we have

$$
\begin{aligned}
& \left\{F_{\text {comb }}(s), F_{p s}(s)\right\} \\
& \sim \frac{K\left(1-\rho_{1}\right)\left(1-\rho_{2}\right)}{\left[1+\rho_{1}(M-1)\right]\left[1+\rho_{2}(M-1)\right]}\left[\frac{\mu_{1} s}{4 \psi_{1} \psi_{2} \bar{\gamma}} \ln (\bar{\gamma})\right]^{M} .
\end{aligned}
$$

${ }^{4}$ In fact, an outage in DF relaying networks depends on the minimum channel coefficient among the source-relay and the relay-destination links. Hence, we can employ the minimum channel coefficient as the equivalent channel quality indicator here. 


\section{VG AF relay selection}

Similarly, the instantaneous end-to-end SNR for VG AF case can be given by [27]

$$
\operatorname{SNR}(m, k)=\frac{\left|h_{1}(m, k)\right|^{2}\left|h_{2}(m, k)\right|^{2} P_{t}^{2}}{\left(\left|h_{1}(m, k)\right|^{2} P_{t}+\left|h_{2}(m, k)\right|^{2} P_{t}+N_{0}\right) N_{0}} .
$$

Again, performing series expansion at $\bar{\gamma} \rightarrow \infty$ yields the asymptotic expression for $F\left(s \mid T_{1}(k), T_{2}(k)\right)$ for VG AF relay systems

$F\left(s \mid T_{1}(k), T_{2}(k)\right) \sim\left[\frac{s}{2}\left(e^{-\frac{\rho_{1} T_{1}(k)}{2 \psi_{1}}} / \psi_{1}+e^{-\frac{\rho_{2} T_{2}(k)}{2 \psi_{2}}} / \psi_{2}\right)\right] \frac{1}{\bar{\gamma}}$.

Proof: See Appendix C.

Obviously, $c_{i_{0}}\left(s \mid T_{1}(k), T_{2}(k)\right)$ for $\mathrm{VG}$ AF relay system is exactly the same as given in (19). Likewise, by (16), the unconditional outage probability in $\mathrm{VG}$ AF relaying network can be determined by

$$
\begin{aligned}
\left\{F_{c o m b}(s), F_{p s}(s)\right\} & \sim K\left(1-\rho_{1}\right)\left(1-\rho_{2}\right)\left(\frac{s}{2 \bar{\gamma}}\right)^{M} \sum_{m=0}^{M}\left(\begin{array}{l}
M \\
m
\end{array}\right) \\
& \times \frac{\left(\frac{1}{\psi_{1}}\right)^{M-m}\left(\frac{1}{\psi_{2}}\right)^{m}}{\left[1+\rho_{1}(M-m-1)\right]\left[1+\rho_{2}(m-1)\right]} .
\end{aligned}
$$

Note, the unconditional outage probability of VG AF relay systems (c.f. (27)) is exactly the same as that given in DF relay systems (c.f. (20)), which aligns with the numerical results presented in [28]. Also, by the definition of the diversity gain

$$
d_{o}=-\lim _{\bar{\gamma} \rightarrow \infty} \frac{\log F_{c o m b}(s)}{\log \bar{\gamma}},
$$

we can see that for all three kinds of relays, the diversity gain is the same value given by the number of relays $M$. That is, the diversity advantage of combined selection will not be shadowed by different forwarding protocols and channel correlations and is only related to the number of available relays.

\section{Asymptotic Outage Performance Analysis With OTHER SELECTIONS}

As we analyzed in the previous section, combined relay selection is powerful in terms of its outstanding outage performance and is able to reduce the system complexity ${ }^{5}$. Furthermore, the equivalence principle can be applied to other selection scenarios with a slight modification of the proposed theorem, e.g. transmit antenna selection and multi-hop branch selection. In this section, two examples are presented to illustrate the feasibility and the expandability of combined selection in other scenarios.

\footnotetext{
${ }^{5}$ To be more specific, the system complexity referred here is related to the number of selected relays, because this number is closely related to the selection and synchronization processes [10]. In addition, combined selection will reduce the number of relays that are accessed for transmission compared to per-subcarrier selection, which will bring an extra system-level efficiency improvement [11].
}

\section{A. Transmit antenna selection with selection combining}

In this subsection, combined transmit antenna selection is analyzed. Before analyzing, it should be clarified that although combined transmit antenna selection has been analyzed in [1], it does not consider channel correlation. Therefore, the combined transmit antenna selection with selection combining over spatially correlated channels is first analyzed in our paper.

A typical MIMO system with combined transmit antenna selection can be illustrated in Fig. 2. Here, $M$ transmit antennas and $N$ receive antennas are considered. Also, the received signal is produced by selection combining at the receiver for each subcarrier. For the convenience purpose, we assume the physical separation among receive antennas is large enough, so that channel correlation only exists at the transmitter. Therefore, we can assume the channel from the $m$ th transmit antenna to the $n$th receive antenna for the $k$ th subcarrier is organized by

$$
\begin{aligned}
h(m, k, n)= & {\left[\sqrt{1-\rho} x(m, k, n)+\sqrt{\rho} x_{0}(k)\right] } \\
& +j\left[\sqrt{1-\rho} y(m, k, n)+\sqrt{\rho} y_{0}(k)\right],
\end{aligned}
$$

where $x(m, k, n), y(m, k, n), x_{0}(k)$ and $y_{0}(k)$ are i.i.d. as $\mathcal{N}(0, \mu / 2) ; \rho$ is the cross-correlation coefficient. Again, we can fix $x_{0}(k)$ and $y_{0}(k)$ and denote $T(k)=X_{0}^{2}(k)+Y_{0}^{2}(k)$, so that $|h(m, k, n)|^{2} \sim \chi_{2}(\sqrt{\rho T(k)}, \mu(1-\rho))$.

Therefore, the average SNR from the $m$ th transmit antenna for the $k$ th subcarrier after selection combining at the receiver is

$$
\operatorname{SNR}(m, k)=\frac{P_{t}}{N_{0}} \max _{1 \leq n \leq N}|h(m, k, n)|^{2} .
$$

Hence, we can determine the CDF by

$$
\begin{aligned}
F(s \mid T(k)) & =\left[1-Q\left(\sqrt{\frac{\rho T(k)}{\psi}}, \sqrt{\frac{s}{\bar{\gamma} \psi}}\right)\right]^{N} \\
& \sim\left[\frac{s}{2 \psi} e^{-\frac{\rho T(k)}{2 \psi}}\right]^{N}\left(\frac{1}{\bar{\gamma}}\right)^{N},
\end{aligned}
$$

where $\psi=\mu(1-\rho) / 2$.

By the combined selection criterion given in (3), we can perform the similar derivation process for MIMO systems to derive

$$
\left\{F_{c o m b}(s), F_{p s}(s)\right\} \sim \frac{K(1-\rho)}{1+\rho(M N-1)}\left(\frac{s}{2 \psi \bar{\gamma}}\right)^{M N} .
$$

Also, by (28) we can obtain the diversity gain in this scenario is $M N$.

\section{B. Multi-hop DF branch selection}

As shown in Fig. 3, a multi-hop DF relay system with branch selection can be illustrated, in which $W$ hops are considered [29]. For the $w$ th hop given $w \in \mathcal{W}=\{1,2, \ldots, W\}$, we can extend the system model constructed for two-hop DF relay system and obtain the conditional distribution of $h_{w}(m, k)$ in terms of $\rho_{w}, \mu_{w}$ and $T_{w}(k)$. Meanwhile, because the outage event in a multi-hop DF system is dominated by the worst channel condition among all hops, we can define the 


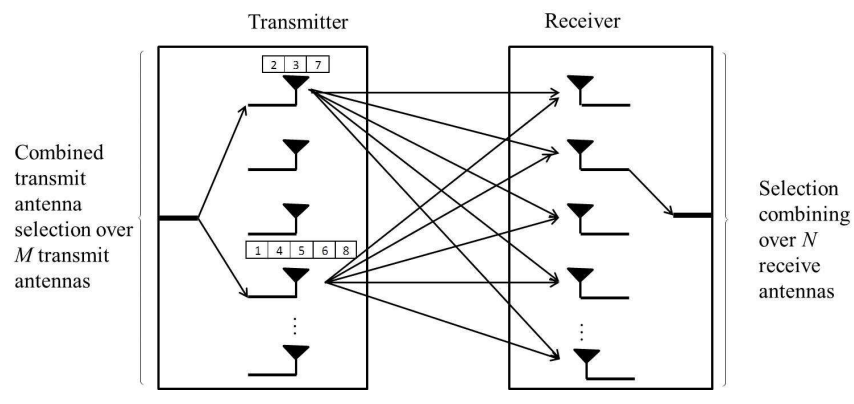

Fig. 2. Illustration of a typical MIMO system with combined transmit antenna selection and selection combining at the receiver, given $K=8$ and $L=2$. The numbers in boxes are corresponding to the sequence numbers of subcarriers.

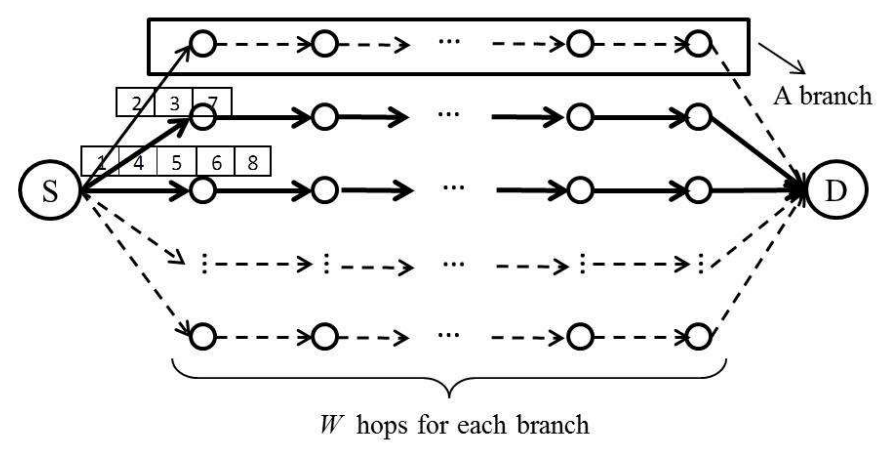

Fig. 3. Illustration of a typical multi-hop system with combined branch selection, given $K=8$ and $L=2$. The numbers in boxes are corresponding to the sequence numbers of subcarriers.

equivalent instantaneous end-to-end SNR for the $m$ th branch and the $k$ th subcarrier by

$$
\operatorname{SNR}(m, k)=\frac{P_{t}}{N_{0}} \min _{\mathcal{W}}\left|h_{w}(m, k)\right|^{2} .
$$

By performing a similar derivation as given previously for the DF relay systems, we have

$$
\begin{aligned}
& F\left(s \mid T_{1}(k), T_{2}(k), \ldots, T_{W}(k)\right) \\
& =1-\prod_{w=1}^{W}\left[1-F_{h_{w}}\left(\frac{s}{\bar{\gamma}} \mid T_{w}(k)\right)\right] \sim\left[\frac{s}{2} \sum_{w=1}^{W} \frac{e^{-\frac{\rho_{w} T_{w}(k)}{2 \psi_{w}}}}{\psi_{w}}\right] \frac{1}{\bar{\gamma}} .
\end{aligned}
$$

Again, applying the combined selection scheme given in (3) with respect to $\operatorname{SNR}(m, k)$, we deduce

$$
\begin{aligned}
& \left\{F_{\text {comb }}(s), F_{p s}(s)\right\} \sim K\left(\frac{s}{2 \bar{\gamma}}\right)^{M} \\
& \times \sum_{\sum_{w=1}^{W} g_{w}=M}\left(\begin{array}{c}
M \\
g_{1}, g_{2}, \ldots, g_{W}
\end{array}\right) \prod_{w=1}^{W} \frac{\left(1-\rho_{w}\right)\left(\frac{1}{\psi_{w}}\right)^{g_{w}}}{1+\rho_{w}\left(g_{w}-1\right)},
\end{aligned}
$$

where $\left\{g_{w}\right\}$ is a set of nonnegative integers satisfying $\sum_{w=1}^{W} g_{w}=M$; the multinomial coefficients $\left(\begin{array}{c}M \\ g_{1}, g_{2}, \ldots, g_{W}\end{array}\right)$ are given by

$$
\left(\begin{array}{c}
M \\
g_{1}, g_{2}, \ldots, g_{W}
\end{array}\right)=\frac{M !}{g_{1} ! g_{2} !, \ldots, g_{W} !} .
$$

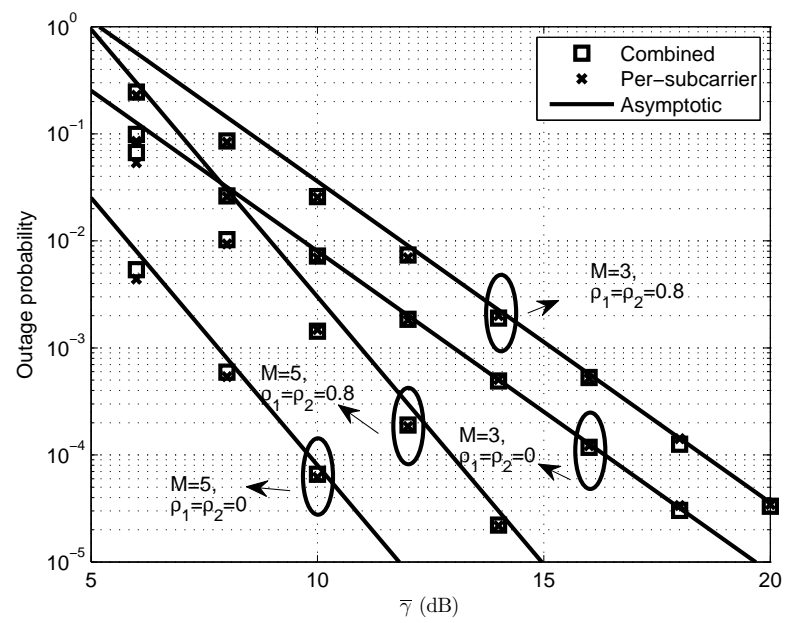

Fig. 4. Two-hop DF relay selection case: outage probability vs. SNR for per-subcarrier and combined bulk/per-subcarrier selection systems.

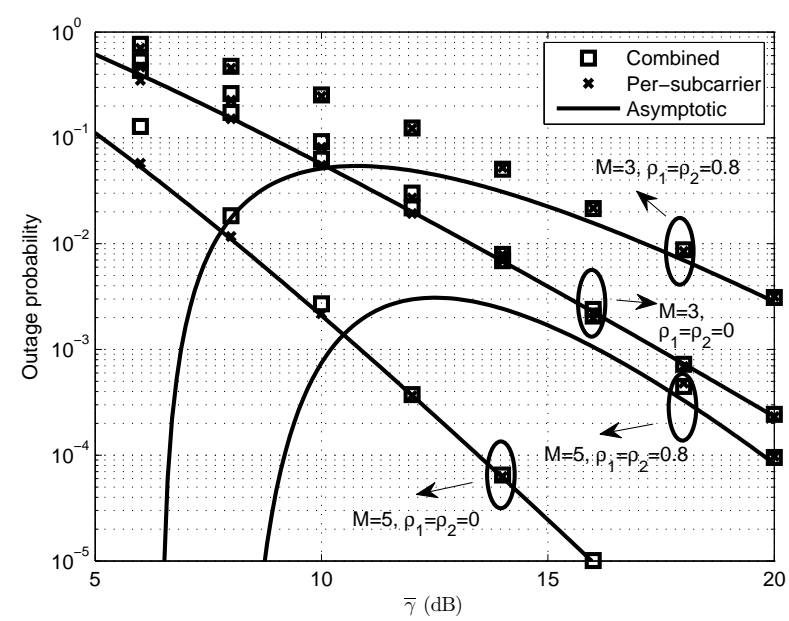

Fig. 5. Two-hop FG AF relay selection case: outage probability vs. SNR for per-subcarrier and combined bulk/per-subcarrier selection systems.

Furthermore, we can derive the diversity gain similarly as above for the DF relay case and obtain $d_{o}=M$ in this multihop case. This indicates that the increase in the number of hops will not affect the diversity gain of a DF forwarding network.

\section{NUMERICAL RESULTS}

First, to verify our analysis in Section III and Section IV, we employ Monte Carlo simulation methods to numerically study the outage performances of OFDM systems employing per-subcarrier and combined selection schemes with three forwarding protocols. Meanwhile, the asymptotic outage performance at high SNR is also taken into account in our simulations. In particular, we let $K=8, s=1$ (i.e. 0 $\mathrm{dB}), \mu_{1}=\mu_{2}=2$, for all simulations. Meanwhile, we vary $M \in\{3,5\}$ and $\rho_{1}=\rho_{2} \in\{0,0.8\}$ to observe the effects of $M$ and $\rho_{i}$ on the outage performance. The simulation results corresponding to the three relay protocols are presented in Fig. 


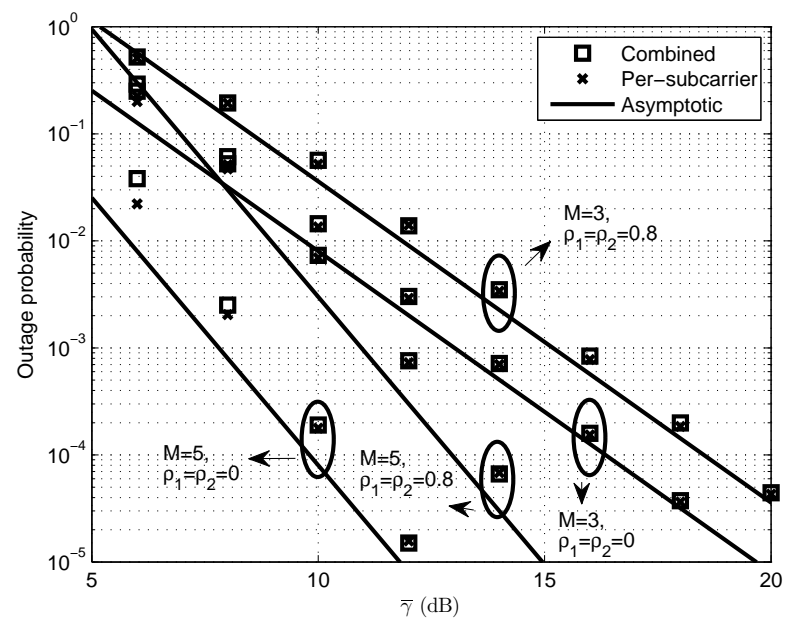

Fig. 6. Two-hop VG AF relay selection case: outage probability vs. SNR for per-subcarrier and combined bulk/per-subcarrier selection systems.

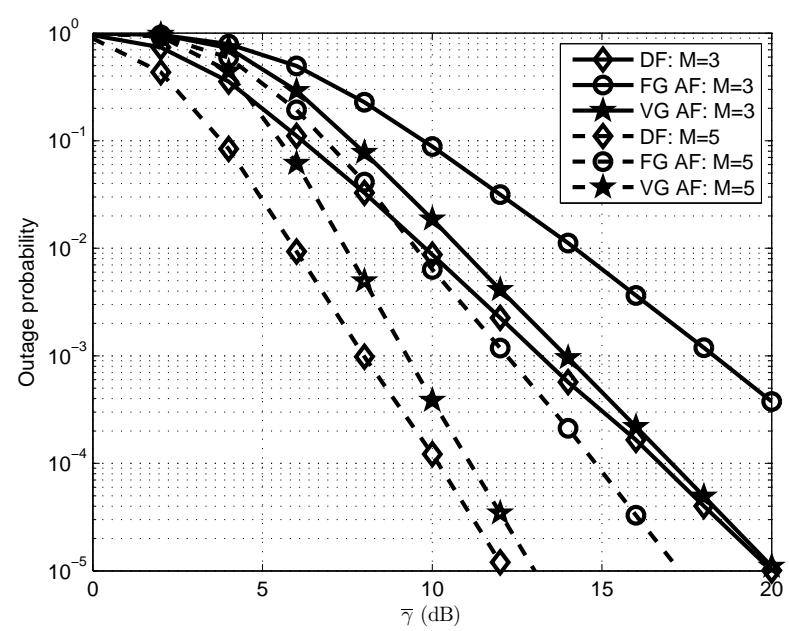

Fig. 7. Outage performances of combined selection corresponding to DF, FG $\mathrm{AF}$ and VG AF relays, given $\rho_{1}=\rho_{2}=0.4$.

4, 5 and 6 , respectively ${ }^{6}$. The outage performance produced by our proposed combined selection scheme is compared with the benchmark outage performance produced by the conventional per-subcarrier selection scheme. Also, the asymptotic curves are given to illustrate the trends of numerical results.

From these three figures, we can summarize some key points with respect to the combined selection scheme. Most importantly, it has been verified that the equivalence principle holds for all three types of relay networks over equally spatially correlated channels. That is, the relay systems employing combined selection can achieve the optimal outage performance as those employing per-subcarrier selection at high SNR. Meanwhile, the increase in the number of relays $M$ will yield a better outage performance, since a larger diversity can be provided. Note, however, that an increase in $M$ does

\footnotetext{
${ }^{6}$ Here, more higher order terms for FG AF case are kept in order to illustrate the convergence between numerical and asymptotic results within a reasonable SNR range.
}

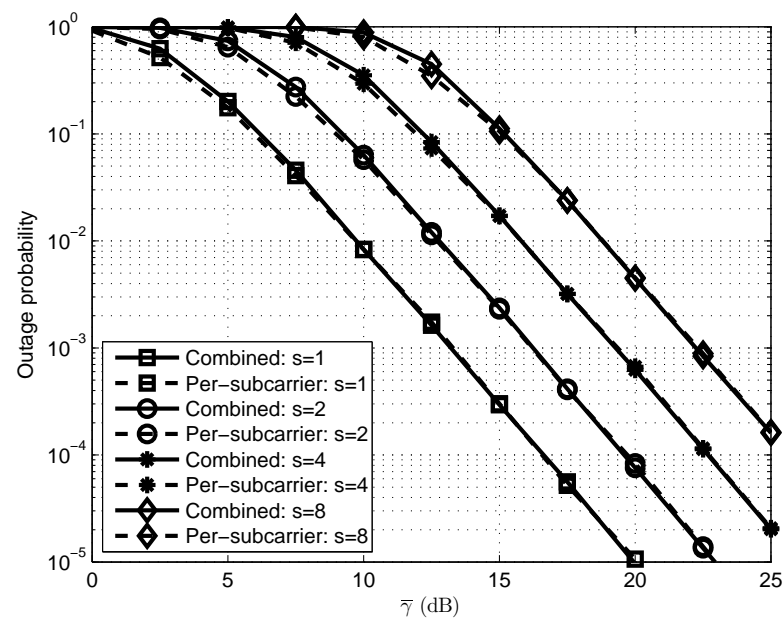

Fig. 8. Outage performances of combined selection corresponding to DF relays, given $s \in\{1,2,4,8\}$.

not indicate that the number of utilized relays increases for the combined selection system since $\left|\mathcal{L}_{\text {comb }}\right|=2$, whereas only the range of selection is enlarged. As for channel correlation, it is apparent that the diversity advantage of cooperative systems brought by relay selection will not be shadowed by channel correlation, as long as $\rho_{1} \rho_{2} \neq 1$. On the other hand, a higher cross-correlation coefficient, whichever in the first hop or second hop, will result in a higher outage probability. In other words, channel correlation has a detrimental impact on the coding gain of a cooperative network. Although we mainly analyze the outage performance in the high SNR region, some important features of combined selection in the low SNR region can also be observed from these figures. First, there exists a gap between combined and per-subcarrier selections, which is caused by the difference in the numbers of selected relays and the fact that fading at low SNR is not dominated by the worst channel. Therefore, a smaller $M$ will lead to a smaller gap at low SNR, and in particular it is expected that the gap will be eliminated when $M=2$. Also, there is not an evident impact by choosing different $\rho_{i}$ on the performance gap at low SNR, because channel correlation does not affect the number of selected relays and thus equivalent to both selection schemes.

In comparison with the outage performances shown in these three figures, we can also have a rough insight into the merits and drawbacks corresponding to three types of relays. First, the outage performances given by DF and VG AF relays are close to each other at high SNR, which aligns with our expectation, because both have the same asymptotic outage performance (c.f. (20) and (27)). And both outage performances are better than the outage performance given by FG AF case. On the other hand, the superiority in outage performance is paid by a higher system complexity. The former needs to decode received signals at relay nodes, and the latter needs to estimate the channel in the first hop by a real-time manner. While the FG AF relay system is relatively simple and retransmits all received signals by a specified and fixed mechanism, 
which only needs to estimate the average channel gain in the first hop once. Therefore, there exists a trade-off between outage performance and system complexity among all three types of relays, which should be considered carefully when implementing cooperative networks. To clearly illustrate the outage performances of these three relays, we also simulate the case $\rho_{1}=\rho_{2}=0.4$ for all three types of relays and plot the results in Fig. 7.

Meanwhile, to reveal the effects of different $s$ on the outage performance, we can take the DF relay case as an example with fixed $M=3$ and $\rho_{1}=\rho_{2}=0.4$. Then, we vary $s \in\{1,2,4,8\}$ and plot the outage performance in Fig. 8. As expected, a larger $s$ will lead to a worse outage performance. In addition, $s$ has an obvious impact on the convergence rate of combined selection to per-subcarrier selection; a larger $s$ indicates a higher $\bar{\gamma}$ is required in order to obtain the equivalent performance.

Furthermore, another two extended cases as analyzed in Section $\mathrm{V}$ are also numerically simulated, and the results are given in Fig. 9 and Fig. 10 respectively. For the case of antenna selection, we keep all configurations as the same as for relay selection, but vary $\rho \in\{0.4,0.8\}$ and let $N=1^{7}$. Meanwhile, for the case of branch-selection, we let $W=3$ and vary $\rho_{1}=\rho_{2}=\rho_{3} \in\{0.4,0.8\}$. From these two figures, the feasibility and the expandability of the equivalence principle is verified. It is obvious that combined selection is not exclusive for antenna selection or relay selection, rather, it is a generic selection algorithm. The equivalence principle can be constructed as long as an OFDM system is given, regardless of the selection nature and channel fading condition. Besides, comparing Fig. 4 and Fig. 10, it is obvious that the increase in the number of hops will yield a poorer performance, since an outage event is a union of the outage in each hop for DF systems.

\section{CONCLUSION}

In conclusion, we proposed a novel relay selection scheme for OFDM systems by combining conventional bulk and persubcarrier selection schemes, and analyzed its outage performance over spatially correlated channels. Specifically, we carried out the asymptotic outage performance analysis of the combined selection scheme in the high SNR region, and proved a generalized theorem stating that if the conditional CDF of the end-to-end SNR, $F\left(s \mid T_{1}(k), T_{2}(k)\right)$, can be expanded as a certain series in the variable $\bar{\gamma}$, combined selection is able to achieve an outage probability equivalent to conventional per-subcarrier selection in the high SNR region. To specify the generalized theorem, we also took three examples of DF, FG AF and VG AF relay systems to analyze and obtained their asymptotic outage probabilities. By Monte Carlo simulations, our analysis was verified by numerical results. The proposed combined relay selection and the proved theorem in this paper provide a general and feasible solution to the

\footnotetext{
${ }^{7}$ The reason why we choose $N=1$ is to reduce the total diversity given by $M N$, so that the convergence between numerical results and asymptotic curve can be shown clearly within a reasonable span of $\bar{\gamma}$ and is thus computationally affordable. In other words, although the selection combining at the receiver is analyzed in Section V, it will not be performed in the numerical simulation.
}

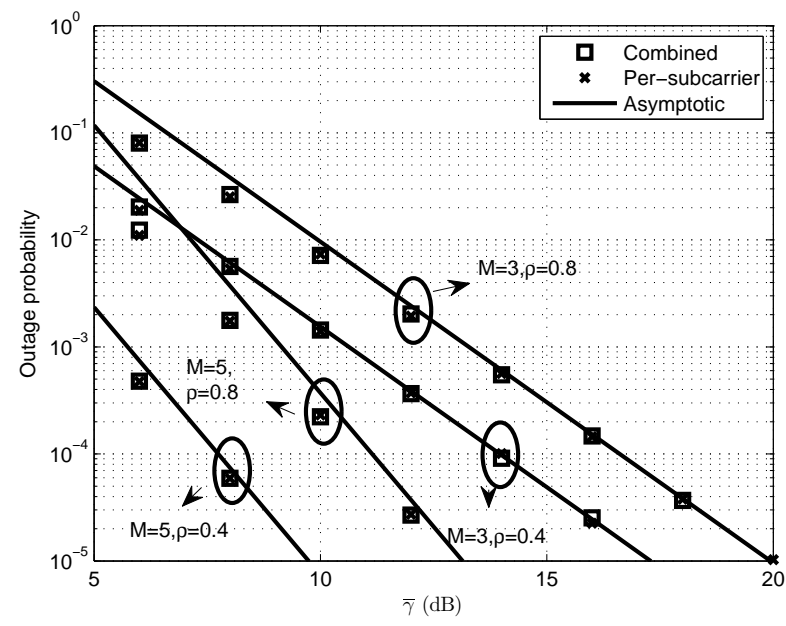

Fig. 9. Transmit antenna selection case: outage probability vs. SNR for persubcarrier and combined bulk/per-subcarrier selection systems, given $N=1$.

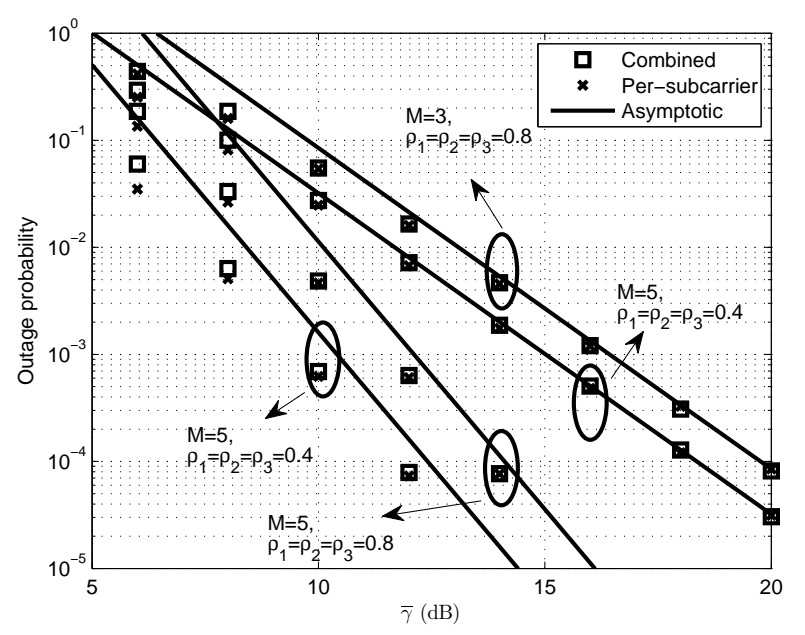

Fig. 10. Multi-hop DF branch selection case: outage probability vs. SNR for per-subcarrier and combined bulk/per-subcarrier selection systems, given $W=3$.

trade-off between system complexity and outage performance when relay selection is applied, so that the optimal outage probability can be achievable at high SNR without using the full set of available relays for selection. Moreover, as shown by two extended applications, the proposed theorem and the generic asymptotic expression for outage probability presented in this paper can also be easily extended to other selections or under other channel fading scenarios, as long as their CDFs of end-to-end SNR is obtainable. In particular, the extension of this theorem to a multi-hop relay selection scenario is still an open issue and is worth investigating comprehensively as a future work. 


\section{APPENDIX A}

DERIVATION OF Asymptotic OUtage Probability of DF NETWORKS

According to (17), we can approximate $F\left(s \mid T_{1}(k), T_{2}(k)\right)$ at high SNR by

$$
\begin{aligned}
& F\left(s \mid T_{1}(k), T_{2}(k)\right)=\mathbb{P}\left\{\frac{P_{t}}{N_{0}} \min \left(\gamma_{1}, \gamma_{2}\right)<s\right\} \\
& =1-\left[1-F_{h_{1}}\left(\frac{s}{\bar{\gamma}} \mid T_{1}(k)\right)\right]\left[1-F_{h_{2}}\left(\frac{s}{\bar{\gamma}} \mid T_{2}(k)\right)\right] \\
& =F_{h_{1}}\left(\frac{s}{\bar{\gamma}} \mid T_{1}(k)\right)+F_{h_{2}}\left(\frac{s}{\bar{\gamma}} \mid T_{2}(k)\right) \\
& -F_{h_{1}}\left(\frac{s}{\bar{\gamma}} \mid T_{1}(k)\right) F_{h_{2}}\left(\frac{s}{\bar{\gamma}} \mid T_{2}(k)\right) \\
& \approx F_{h_{1}}\left(\frac{s}{\bar{\gamma}} \mid T_{1}(k)\right)+F_{h_{2}}\left(\frac{s}{\bar{\gamma}} \mid T_{2}(k)\right) .
\end{aligned}
$$

By (9), we can further obtain

$$
F\left(s \mid T_{1}(k), T_{2}(k)\right)=\sum_{i=1}^{2}\left[1-Q\left(\sqrt{\frac{\rho_{i} T_{i}(k)}{\psi_{i}}}, \sqrt{\frac{s}{\psi_{i} \bar{\gamma}}}\right)\right] .
$$

[23]

Also, we can express $Q\left(\sqrt{\rho_{i} T_{i}(k) / \psi_{i}}, \sqrt{s /\left(\psi_{i} \bar{\gamma}\right)}\right)$ by

$$
\begin{aligned}
& Q\left(\sqrt{\frac{\rho_{i} T_{i}(k)}{\psi_{i}}}, \sqrt{\frac{s}{\psi_{i} \bar{\gamma}}}\right) \\
& =e^{-\frac{\rho_{i} T_{i}(k)}{2 \psi_{i}}} e^{-\frac{s}{2 \psi_{1} \bar{\gamma}}} \sum_{p=0}^{\infty}\left(\sqrt{\frac{\rho_{i} T_{i}(k) \bar{\gamma}}{s}}\right)^{p} I_{p}\left(\frac{1}{\psi_{i}} \sqrt{\frac{\rho_{i} T_{i}(k) s}{\bar{\gamma}}}\right) .
\end{aligned}
$$

Also, we can expand the $p$ th order modified Bessel function of the first kind by [23]

$$
\begin{aligned}
& I_{p}\left(\frac{1}{\psi_{i}} \sqrt{\frac{\rho_{i} T_{i}(k) s}{\bar{\gamma}}}\right) \\
& =\left(\frac{1}{2 \psi_{i}} \sqrt{\frac{\rho_{i} T_{i}(k) s}{\bar{\gamma}}}\right)^{p} \sum_{q=0}^{\infty} \frac{\left(\frac{\rho_{i} T_{i}(k) s}{4 \psi_{i}^{2} \bar{\gamma}}\right)^{q}}{q ! \Gamma(q+p+1)},
\end{aligned}
$$

where $\Gamma(\cdot)$ is the Gamma function.

Therefore, the summation part can be alternatively expressed by

$$
\begin{aligned}
& \sum_{p=0}^{\infty}\left(\sqrt{\frac{\rho_{i} T_{i}(k) \bar{\gamma}}{s}}\right)^{p} I_{p}\left(\frac{1}{\psi_{i}} \sqrt{\frac{\rho_{i} T_{i}(k) s}{\bar{\gamma}}}\right) \\
& =\sum_{q=0}^{\infty} \sum_{p=0}^{\infty} \frac{\left(\frac{\rho_{i} T_{i}(k)}{2 \psi_{i}}\right)^{p}\left(\frac{\rho_{i} T_{i}(k) s}{4 \psi_{i}^{2} \bar{\gamma}}\right)^{q}}{q ! \Gamma(q+p+1)} .
\end{aligned}
$$

Meanwhile, $e^{-\frac{s}{2 \psi_{i} \bar{\gamma}}}$ can also be expanded in terms of $\bar{\gamma}$ by [30]

$$
e^{-\frac{s}{2 \psi_{i} \bar{\gamma}}}=\sum_{p=0}^{\infty} \frac{1}{p !}\left(-\frac{s}{2 \psi_{i} \bar{\gamma}}\right)^{p}
$$

Therefore, substituting (41) and (42) into (39) yields

$$
\begin{aligned}
& Q\left(\sqrt{\frac{\rho_{i} T_{i}(k)}{\psi_{i}}}, \sqrt{\frac{s}{\psi_{i} \bar{\gamma}}}\right)=e^{-\frac{\rho_{i} T_{i}(k)}{2 \psi_{i}}}\left[\sum_{p=0}^{\infty} \frac{1}{p !}\left(-\frac{s}{2 \psi_{i} \bar{\gamma}}\right)^{p}\right] \\
& \times\left[\sum_{q=0}^{\infty} \sum_{p=0}^{\infty} \frac{\left(\frac{\rho_{i} T_{i}(k)}{2 \psi_{i}}\right)^{p}\left(\frac{\rho_{i} T_{i}(k) s}{4 \psi_{i}^{2} \bar{\gamma}}\right)^{q}}{q ! \Gamma(q+p+1)}\right] \sim 1-e^{-\frac{\rho_{i} T_{i}(k)}{2 \psi_{i}}} \frac{s}{2 \psi_{i} \bar{\gamma}} .
\end{aligned}
$$

Subsequently, substituting (43) into (38) yields the asymptotic expression for $F\left(s \mid T_{1}(k), T_{2}(k)\right)$ at $\bar{\gamma} \rightarrow \infty$

$$
F\left(s \mid T_{1}(k), T_{2}(k)\right) \sim\left[\frac{s}{2}\left(e^{-\frac{\rho_{1} T_{1}(k)}{2 \psi_{1}}} / \psi_{1}+e^{-\frac{\rho_{2} T_{2}(k)}{2 \psi_{2}}} / \psi_{2}\right)\right] \frac{1}{\bar{\gamma}} .
$$

\section{APPENDIX B}

\section{Derivation of As YMPtotic OUtage Probability of FG AF NETWORKS}

We can adopt a similar method as proposed in [31] to derive the CDF of end-to-end SNR over correlated channels and determine $F\left(s \mid T_{1}(k), T_{2}(k)\right)$ by

$$
\begin{aligned}
& F\left(s \mid T_{1}(k), T_{2}(k)\right)=\mathbb{P}\left\{\frac{\gamma_{1} \gamma_{2} P_{t}^{2}}{\left(\mu_{1} P_{t}+\gamma_{2} P_{t}+N_{0}\right) N_{0}}<s\right\} \\
& =\mathbb{P}\left\{\gamma_{1}<\frac{s\left(\mu_{1}+\gamma_{2}+\frac{1}{\bar{\gamma}}\right)}{\gamma_{2} \bar{\gamma}}\right\} \\
& =\int_{0}^{\infty} F_{h_{1}}\left(\frac{s\left(\mu_{1}+\gamma_{2}+\frac{1}{\bar{\gamma}}\right)}{\gamma_{2} \bar{\gamma}} \mid T_{1}(k)\right) f_{h_{2}}\left(\gamma_{2} \mid T_{2}(k)\right) \mathrm{d} \gamma_{2} .
\end{aligned}
$$

Considering $\bar{\gamma} \rightarrow \infty$, we can approximate the outage condition by omitting the higher order terms corresponding to $1 / \bar{\gamma}$ and obtain

$$
\frac{s\left(\mu_{1}+\gamma_{2}+\frac{1}{\bar{\gamma}}\right)}{\gamma_{2} \bar{\gamma}} \approx \frac{s\left(\mu_{1}+\gamma_{2}\right)}{\gamma_{2} \bar{\gamma}},
$$

and thereby have

$$
\begin{aligned}
& F\left(s \mid T_{1}(k), T_{2}(k)\right) \\
& \approx \int_{0}^{\infty} F_{h_{1}}\left(\frac{s\left(\gamma_{2}+\mu_{1}\right)}{\gamma_{2} \bar{\gamma}} \mid T_{1}(k)\right) f_{h_{2}}\left(\gamma_{2} \mid T_{2}(k)\right) \mathrm{d} \gamma_{2} .
\end{aligned}
$$

Now, let us take a close look at the conditional CDF $F_{h_{1}}\left(\frac{s\left(\gamma_{2}+\mu_{1}\right)}{\gamma_{2} \bar{\gamma}} \mid T_{1}(k)\right)$. Again, by (9), we can express this conditional $\mathrm{CDF}$ by

$$
\begin{aligned}
& F_{h_{1}}\left(\frac{s\left(\gamma_{2}+\mu_{1}\right)}{\gamma_{2} \bar{\gamma}} \mid T_{1}(k)\right) \\
& =1-Q\left(\sqrt{\frac{\rho_{1} T_{1}(k)}{\psi_{1}}}, \sqrt{\frac{s\left(\gamma_{2}+\mu_{1}\right)}{\psi_{1} \gamma_{2} \bar{\gamma}}}\right) .
\end{aligned}
$$


Therefore, we can perform the similar derivation as we do for DF case and the Marcum Q function can be alternatively expressed as

$$
\begin{aligned}
& Q\left(\sqrt{\frac{\rho_{1} T_{1}(k)}{\psi_{1}}}, \sqrt{\left.\frac{s\left(\gamma_{2}+\mu_{1}\right)}{\psi_{1} \gamma_{2} \bar{\gamma}}\right)}\right. \\
& =e^{-\frac{\rho_{1} T_{1}(k)}{2 \psi_{1}}} \sum_{q=0}^{\infty} \sum_{p=0}^{\infty} \frac{\left(\frac{\rho_{1} T_{1}(k)}{2 \psi_{1}}\right)^{p}\left(\frac{\rho_{1} T_{1}(k)}{4 \psi_{1}^{2}}\right)^{q}}{q ! \Gamma(q+p+1)} \\
& \times\left[\frac{s\left(\gamma_{2}+\mu_{1}\right)}{\bar{\gamma}}\right]^{q}\left(\frac{1}{\gamma_{2}}\right)^{q} e^{-\frac{s\left(\gamma_{2}+\mu_{1}\right)}{2 \psi_{1} \gamma_{2} \bar{\gamma}}} .
\end{aligned}
$$

Meanwhile, we can also expand $f_{h_{2}}\left(\gamma_{2} \mid T_{2}(k)\right)$ by [23]

$$
f_{h_{2}}\left(\gamma_{2} \mid T_{2}(k)\right)=\frac{1}{2 \psi_{2}} e^{-\frac{\rho_{2} T_{2}(k)}{2 \psi_{2}}} e^{-\frac{\gamma_{2}}{2 \psi_{2}}} \sum_{u=0}^{\infty} \frac{\left(\frac{\rho_{2} T_{2}(k) \gamma_{2}}{4 \psi_{2}^{2}}\right)^{u}}{(u !)^{2}} .
$$

Therefore, we can obtain

$$
\begin{aligned}
& \int_{0}^{\infty} Q\left(\sqrt{\frac{\rho_{1} T_{1}(k)}{\psi_{1}}}, \sqrt{\frac{s\left(\gamma_{2}+\mu_{1}\right)}{\psi_{1} \gamma_{2} \bar{\gamma}}}\right) f_{h_{2}}\left(\gamma_{2} \mid T_{2}(k)\right) \mathrm{d} \gamma_{2} \\
& =\frac{1}{2 \psi_{2}} e^{-\frac{\rho_{1} T_{1}(k)}{2 \psi_{1}}} e^{-\frac{\rho_{2} T_{2}(k)}{2 \psi_{2}}} \sum_{q=0}^{\infty} \sum_{p=0}^{\infty} \sum_{u=0}^{\infty} \mathcal{C}(q, p, u) \mathcal{H}(q, u),
\end{aligned}
$$

where

$$
\mathcal{C}(q, p, u)=\frac{\left(\frac{\rho_{1} T_{1}(k)}{2 \psi_{1}}\right)^{p}\left(\frac{\rho_{1} T_{1}(k)}{2 \psi_{1}}\right)^{q}\left(\frac{\rho_{2} T_{2}(k)}{2 \psi_{2}}\right)^{u}}{q ! \Gamma(q+p+1)(u !)^{2}}
$$

and

$\mathcal{H}(q, u)=\int_{0}^{\infty}\left[\frac{s\left(\gamma_{2}+\mu_{1}\right)}{2 \psi_{1} \gamma_{2} \bar{\gamma}}\right]^{q}\left(\frac{\gamma_{2}}{2 \psi_{2}}\right)^{u} e^{-\frac{s\left(\gamma_{2}+\mu_{1}\right)}{2 \psi_{1} \gamma_{2} \bar{\gamma}}} e^{-\frac{\gamma_{2}}{2 \psi_{2}}} \mathrm{~d} \gamma_{2}$.

Because we are only interested in the high SNR region, i.e. $\bar{\gamma} \rightarrow \infty$, we can approximate (51) by considering $q=0$ and $q=1$ only:

$$
\begin{aligned}
& \int_{0}^{\infty} Q\left(\sqrt{\frac{\rho_{1} T_{1}(k)}{\psi_{1}}}, \sqrt{\left.\frac{s\left(\gamma_{2}+\mu_{1}\right)}{\psi_{1} \gamma_{2} \bar{\gamma}}\right) f_{h_{2}}\left(\gamma_{2} \mid T_{2}(k)\right) \mathrm{d} \gamma_{2}}\right. \\
& \approx \frac{1}{2 \psi_{2}} e^{-\frac{\rho_{1} T_{1}(k)}{2 \psi_{1}}} e^{-\frac{\rho_{2} T_{2}(k)}{2 \psi_{2}}} \sum_{u=0}^{\infty}\left[\sum_{p=0}^{\infty} \mathcal{C}(0, p, u)\right] \mathcal{H}(0, u) \\
& +\frac{1}{2 \psi_{2}} e^{-\frac{\rho_{1} T_{1}(k)}{2 \psi_{1}}} e^{-\frac{\rho_{2} T_{2}(k)}{2 \psi_{2}}} \sum_{u=0}^{\infty}\left[\sum_{p=0}^{\infty} \mathcal{C}(1, p, u)\right] \mathcal{H}(1, u)
\end{aligned}
$$

Then, the closed-form expressions for $\mathcal{H}(0, u)$ and $\mathcal{H}(1, u)$ are now obtainable and given by

$$
\mathcal{H}(0, u)=4 \psi_{2} e^{-\frac{s}{2 \psi_{1} \bar{\gamma}}}\left(\frac{\mu_{1} s}{4 \psi_{1} \psi_{2} \bar{\gamma}}\right)^{\frac{u+1}{2}} K_{u+1}\left(\sqrt{\frac{\mu_{1} s}{\psi_{1} \psi_{2} \bar{\gamma}}}\right)
$$

and

$$
\begin{aligned}
& \mathcal{H}(1, u)=2 \psi_{2} e^{-\frac{s}{2 \psi_{1} \bar{\gamma}}}\left(\frac{s}{\psi_{1} \bar{\gamma}}\right)\left(\frac{\mu_{1} s}{4 \psi_{1} \psi_{2} \bar{\gamma}}\right)^{\frac{u+1}{2}} K_{u+1}\left(\sqrt{\frac{\mu_{1} s}{\psi_{1} \psi_{2} \bar{\gamma}}}\right) \\
& +e^{-\frac{s}{2 \psi_{1} \bar{\gamma}}}\left(\frac{\mu_{1} s}{\psi_{1} \bar{\gamma}}\right)\left(\frac{\mu_{1} s}{4 \psi_{1} \psi_{2} \bar{\gamma}}\right)^{\frac{u}{2}} K_{u}\left(\sqrt{\frac{\mu_{1} s}{\psi_{1} \psi_{2} \bar{\gamma}}}\right)
\end{aligned}
$$

where $K_{v}(\cdot)$ is the $v$ th order modified Bessel function of the second kind.

Meanwhile, we can also obtain

$$
\sum_{p=0}^{\infty} \mathcal{C}(0, p, u)=\frac{\left(\frac{\rho_{2} T_{2}(k)}{2 \psi_{2}}\right)^{u} e^{\frac{\rho_{1} T_{1}(k)}{2 \psi_{2}}}}{(u !)^{2}}
$$

and

$$
\sum_{p=0}^{\infty} \mathcal{C}(1, p, u)=\frac{\left(\frac{\rho_{2} T_{2}(k)}{2 \psi_{2}}\right)^{u}\left(e^{\frac{\rho_{1} T_{1}(k)}{2 \psi_{2}}}-1\right)}{(u !)^{2}}
$$

Now, we can perform series expansion at $\bar{\gamma} \rightarrow \infty$ and obtain the asymptotic expressions for (51) by

$$
\begin{aligned}
& \int_{0}^{\infty} Q\left(\sqrt{\frac{\rho_{1} T_{1}(k)}{\psi_{1}}}, \sqrt{\frac{s\left(\gamma_{2}+\mu_{1}\right)}{\psi_{1} \gamma_{2} \bar{\gamma}}}\right) f_{h_{2}}\left(\gamma_{2} \mid T_{2}(k)\right) \mathrm{d} \gamma_{2} \\
& \sim 1-\left(\frac{\mu_{1} s}{4 \psi_{1} \psi_{2}} e^{-\frac{\rho_{1} T_{1}(k)}{2 \psi_{1}}} e^{-\frac{\rho_{2} T_{2}(k)}{2 \psi_{2}}}\right) \frac{\ln (\bar{\gamma})}{\bar{\gamma}}
\end{aligned}
$$

Also, due to the property of a PDF, it is obvious that $\int_{0}^{\infty} f_{h_{2}}\left(\gamma_{2} \mid T_{2}(k)\right) \mathrm{d} \gamma_{2}=1$. As a result, by (48) and (59) the asymptotic expression for (47) can be given by

$$
F\left(s \mid T_{1}(k), T_{2}(k)\right) \sim\left(\frac{\mu_{1} s}{4 \psi_{1} \psi_{2}} e^{-\frac{\rho_{1} T_{1}(k)}{2 \psi_{1}}} e^{-\frac{\rho_{2} T_{2}(k)}{2 \psi_{2}}}\right) \frac{\ln (\bar{\gamma})}{\bar{\gamma}} .
$$

\section{APPENDIX C}

Derivation of As ymptotic Outage Probability of VG AF NETWORKS

Similar to the analysis of the FG AF case, we can express $F\left(s \mid T_{1}(k), T_{2}(k)\right)$ by

$$
\begin{aligned}
F & \left(s \mid T_{1}(k), T_{2}(k)\right)=\mathbb{P}\left\{\frac{\gamma_{1} \gamma_{2} P_{t}^{2}}{\left(\gamma_{1} P_{t}+\gamma_{2} P_{t}+N_{0}\right) N_{0}}<s\right\} \\
= & \mathbb{P}\left\{\gamma_{1}<\frac{s\left(\gamma_{2}+\frac{1}{\bar{\gamma}}\right)}{\gamma_{2} \bar{\gamma}-s} \mid \gamma_{2}>\frac{s}{\bar{\gamma}}\right\} \\
& +\mathbb{P}\left\{\gamma_{1}>\frac{s\left(\gamma_{2}+\frac{1}{\bar{\gamma}}\right)}{\gamma_{2} \bar{\gamma}-s} \mid 0<\gamma_{2}<\frac{s}{\bar{\gamma}}\right\} \\
= & \int_{\frac{s}{\bar{\gamma}}}^{\infty} F_{h_{1}}\left(\frac{s\left(\gamma_{2}+\frac{1}{\bar{\gamma}}\right)}{\gamma_{2} \bar{\gamma}-s} \mid T_{1}(k)\right) f_{h_{2}}\left(\gamma_{2} \mid T_{2}(k)\right) \mathrm{d} \gamma_{2}+ \\
& \int_{0}^{\frac{s}{\bar{\gamma}}}\left[1-F_{h_{1}}\left(\frac{s\left(\gamma_{2}+\frac{1}{\bar{\gamma}}\right)}{\gamma_{2} \bar{\gamma}-s} \mid T_{1}(k)\right)\right] f_{h_{2}}\left(\gamma_{2} \mid T_{2}(k)\right) \mathrm{d} \gamma_{2} .
\end{aligned}
$$


Again, we first need to expand $s\left(\gamma_{2}+1 / \bar{\gamma}\right) /\left(\gamma_{2} \bar{\gamma}-s\right)$ at $\bar{\gamma} \rightarrow \infty$ in order to derive the asymptotic expression. Here we have

$$
\frac{s\left(\gamma_{2}+\frac{1}{\bar{\gamma}}\right)}{\gamma_{2} \bar{\gamma}-s} \approx \frac{s}{\bar{\gamma}} .
$$

Therefore, we can approximate $F\left(s \mid T_{1}(k), T_{2}(k)\right)$ at high SNR by

$$
\begin{aligned}
F & \left(s \mid T_{1}(k), T_{2}(k)\right) \\
\approx & \int_{\frac{s}{\bar{\gamma}}}^{\infty} F_{h_{1}}\left(\frac{s}{\bar{\gamma}} \mid T_{1}(k)\right) f_{h_{2}}\left(\gamma_{2} \mid T_{2}(k)\right) \mathrm{d} \gamma_{2} \\
& +\int_{0}^{\frac{s}{\bar{\gamma}}}\left[1-F_{h_{1}}\left(\frac{s}{\bar{\gamma}} \mid T_{1}(k)\right)\right] f_{h_{2}}\left(\gamma_{2} \mid T_{2}(k)\right) \mathrm{d} \gamma_{2} \\
= & F_{h_{1}}\left(\frac{s}{\bar{\gamma}} \mid T_{1}(k)\right)+F_{h_{2}}\left(\frac{s}{\bar{\gamma}} \mid T_{1}(k)\right) \\
& -2 F_{h_{1}}\left(\frac{s}{\bar{\gamma}} \mid T_{1}(k)\right) F_{h_{2}}\left(\frac{s}{\bar{\gamma}} \mid T_{1}(k)\right) \\
\approx & F_{h_{1}}\left(\frac{s}{\bar{\gamma}} \mid T_{1}(k)\right)+F_{h_{2}}\left(\frac{s}{\bar{\gamma}} \mid T_{1}(k)\right) .
\end{aligned}
$$

Then, all analysis follows the case derived for DF relay and the asymptotic expression is given by the same one in (44).

\section{ACKNOWLEDGMENT}

The authors would like to thank the editor and the anonymous reviewers for their constructive comments which help improve the quality of this paper.

\section{REFERENCES}

[1] J. Coon and M. Sandell, "Combined bulk and per-tone transmit antenna selection in OFDM systems," IEEE Communications Letters, vol. 14, no. 5, pp. 426-428, May 2010.

[2] J. Laneman, D. Tse, and G. W. Wornell, "Cooperative diversity in wireless networks: efficient protocols and outage behavior," IEEE Transactions on Information Theory, vol. 50, no. 12, pp. 3062-3080, Dec. 2004.

[3] A. Chandra, C. Bose, and M. Bose, "Wireless relays for next generation broadband networks," IEEE Potentials, vol. 30, no. 2, pp. 39-43, Mar. 2011.

[4] G. Chen and J. A. Chambers, "Exact outage probability analysis for cooperative af relay network with relay selection in presence of intercell interference," Electronics Letters, vol. 48, no. 21, pp. 1346-1347, Oct. 2012

[5] P. Guo, Y. Bai, Z. Ma, S. Wu, and S. Dang, "Relay technology for multicarrier systems: a research overview," in Proc. IEEE C3IT, Hooghly, India, Feb. 2015.

[6] G. Chen, O. Alnatouh, and J. Chambers, "Two-and four-relay selection schemes for application in interference limited legacy networks," EURASIP Journal on Wireless Communications and Networking, vol. 2012, no. 1, pp. 1-10, 2012.

[7] G. Chen and J. Chambers, "Outage probability in distributed transmission based on best relay pair selection," IET Communications, vol. 6 , no. 12, pp. 1829-1836, Aug. 2012.

[8] G. Chen, O. Alnatouh, and J. Chambers, "Outage probability analysis for a cognitive amplify-and-forward relay network with single and multirelay selection," IET Communications, vol. 7, no. 17, pp. 1974-1981, Nov. 2013.

[9] S. Dang, J. P. Coon, and D. E. Simmons, "Combined bulk and per-tone relay selection in super dense wireless networks," in Proc. IEEE ICC, London, UK, June 2015.

[10] Y. Li, W. Wang, and F.-C. Zheng, "Combined bulk and per-tone relay selection in cooperative OFDM systems," in Proc. IEEE ICCC, Beijing, China, Aug. 2012.
[11] S. Dang, D. E. Simmons, and J. P. Coon, "Comparison of multicarrier relay selection schemes in super dense networks," in Proc. IEEE CAMAD, Guildford, UK, Sept. 2015.

[12] B. Nguyen, R. Afolabi, and K. Kim, "Dependence of outage probability of cooperative systems with single relay selection on channel correlation," IEEE Communications Letters, vol. 17, no. 11, pp. 2060-2063, Nov. 2013.

[13] Q. Zhang and H. Lu, "A general analytical approach to multi-branch selection combining over various spatially correlated fading channels," IEEE Transactions on Communications, vol. 50, no. 7, pp. 1066-1073, Jul. 2002.

[14] R. Swaminathan, R. Roy, and M. Selvaraj, "Performance analysis of triple correlated selection combining for cooperative diversity systems," in Proc. IEEE ICC, Budapest, Hungary, June 2013.

[15] Y. Chen and C. Tellambura, "Distribution functions of selection combiner output in equally correlated Rayleigh, Rician, and Nakagami$\mathrm{m}$ fading channels," IEEE Transactions on Communications, vol. 52, no. 11, pp. 1948-1956, Nov. 2004.

[16] Y. Chau and K.-T. Huang, "Performance of cooperative diversity on correlated dual-hop channels with an amplify-and-forward relay over Rayleigh fading environments," in Proc. IEEE TENCON, Bali, Indonisia, Nov. 2011.

[17] W. S. Kim, W. J. Kim, H. S. Lee, I. M. Kim, D. I. Kim, S. Kim, and K. Lim, "Relay selection in multiple clustered relay networks," in IEEE WTS, New York, US, Apr. 2015.

[18] S. Vakil, M. Dong, and B. Liang, "Effect of cluster size selection on the throughput of multi-hop cooperative relay," in Proc. IEEE VTC Fall, Ottawa, Canada, Sept. 2010.

[19] J. pyo Hong and W. Choi, "Throughput characteristics by multiuser diversity in a cognitive radio system," IEEE Transactions on Signal Processing, vol. 59, no. 8, pp. 3749-3763, Aug. 2011.

[20] H. Nam, Y. chai Ko, and B. Womack, "Performance analysis of OTMRC over i.i.d. Nakagami and non-i.i.d. Rayleigh fading channels," IEEE Transactions on Vehicular Technology, vol. 55, no. 6, pp. 19411946, Nov. 2006

[21] A. Ghasemi and E. Sousa, "Fundamental limits of spectrum-sharing in fading environments," IEEE Transactions on Wireless Communications, vol. 6, no. 2, pp. 649-658, Feb. 2007.

[22] K. Hamdi, W. Zhang, and K. Ben Letaief, "Power control in cognitive radio systems based on spectrum sensing side information," in Proc. IEEE ICC, Glasgow, UK, June 2007.

[23] J. Proakis and M. Salehi, Digital Communications. McGraw-Hill Education, 2007.

[24] D. Tse and P. Viswanath, Fundamentals of Wireless Communication. New York, NY, USA: Cambridge University Press, 2005.

[25] H. David and H. Nagaraja, Order Statistics. Wiley, 2004.

[26] W. Yang and Y. Cai, "Distributed relay selection in OFDM-based cooperative wireless networks," in Proc. IEEE WiCOM, Chengdu, China, Sept. 2010.

[27] D. Simmons, D. Halls, and J. Coon, "OFDM-based nonlinear fixed-gain amplify-and-forward relay systems: SER optimization and experimental testing," in Proc. IEEE EuCNC, Bologna, Italy, June 2014.

[28] G. Huang, Y. Wang, and J. Coon, "Performance of multihop decodeand-forward and amplify-and-forward relay networks with channel estimation," in Proc. IEEE PacRim, Victoria, BC, Aug. 2011.

[29] M. Bhatnagar, R. Mallik, and O. Tirkkonen, "Performance evaluation of best path selection in a multi-hop decode-and-forward cooperative system," IEEE Transactions on Vehicular Technology, to be accepted, 2015.

[30] M. Abramowitz and I. Stegun, Handbook of Mathematical Functions: With Formulas, Graphs, and Mathematical Tables. Dover Publications, 1964.

[31] M. Hasna and M.-S. Alouini, "A performance study of dual-hop transmissions with fixed gain relays," IEEE Transactions on Wireless Communications, vol. 3, no. 6, pp. 1963-1968, Nov. 2004. 


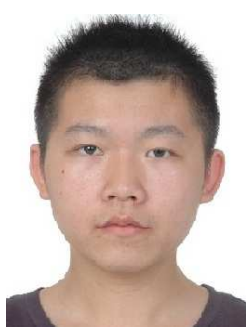

Shuping Dang (S'13) received the B.Eng (Hons) degree in Electrical and Electronic Engineering from the University of Manchester (with first class honors) and the B.Eng in Electrical Engineering and Automation from Beijing Jiaotong University in 2014. He was also a Certified LabVIEW Associate Developer (CLAD) by National Instrument (NI). Mr. Dang is currently a D.Phil candidate with the Department of Engineering Science, University of Oxford. His current research interests include cooperative communication system design. communications, wireless signal processing and 5G

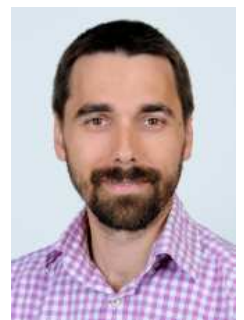

Justin P. Coon (S'02-M'05-SM'10) received a BSc. degree (with distinction) in electrical engineering from the Calhoun Honours College, Clemson University, USA and a Ph.D in communications from the University of Bristol, UK in 2000 and 2005, respectively. In 2004, he joined Toshiba Research Europe Ltd. (TREL) as a Research Engineer working in its Bristol based Telecommunications Research Laboratory (TRL), where he conducted research on a broad range of communication technologies and theories, including single and multi-carrier modulation techniques, estimation and detection, diversity methods, system performance analysis and networks. He held the position of Research Manager from 20102013, during which time he led all theoretical and applied research on the physical layer at TRL. Dr Coon was a Visiting Fellow with the School of Mathematics at the University of Bristol from 2010-2012, and held a position as Reader in the Department of Electrical and Electronic Engineering at the same university from 2012-2013. He joined the University of Oxford in 2013 where he is currently an Associate Professor with the Department of Engineering Science and a Tutorial Fellow of Oriel College.

Dr Coon is the recipient of TRL's Distinguished Research Award for his work on block-spread CDMA, aspects of which have been adopted as mandatory features in the 3GPP LTE Rel-8 standard. He is also a co-recipient of two 'best paper' awards for work presented at ISWCS ' 13 and EuCNC '14. Dr Coon has published in excess of 100 papers in leading international journals and conferences, and is a named inventor on more than 30 patents. He is the Technical Manager of the EU FP7 project DIWINE, has served as an Editor for IEEE TRANSACTIONS ON WIRELESS COMMUNICATIONS (2007 2013), IEEE TRANSACTIONS ON VehICULAR TECHNOLOGY (2013 - 2016; received award for outstanding contribution in 2014) and IEEE WIRELESS COMMUNiCATIONS LetTERS (2016 - present). Dr Coon's research interests include communication theory, information theory and network theory.

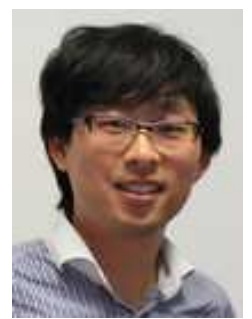

Gaojie Chen (S'09-M'12) received the B. Eng. and B. Ec. in Electrical Information Engineering and International Economics and Trade from the Northwest University, Shaanxi, China, in 2006, and the M.Sc (Distinction) and Ph.D degrees from Loughborough University, Loughborough, UK, in 2008 and 2012, respectively, all in Electrical and Electronic Engineering. From 2008 to 2009 he worked, as a software engineering in DTmobile, Beijing, China, and from 2012 to 2013 as a Research Associate in the School of Electronic, Electrical and Systems Engineering at the Loughborough University, Loughborough, UK. Then he was a Research Fellow with the 5GIC, the Faculty of Engineering and Physical Sciences, University of Surrey, U.K., from 2014 to 2015. He is currently a Research Associate with the Department of Engineering Science, University of Oxford, U.K. His current research interests include information theory, wireless communications, cooperative communications, cognitive radio, secrecy communication and random geometric networks. 